\title{
Maciej RUDNICKI*
}

\section{Les instruments juridiques et financiers du développement écologique en Pologne}

Les instruments juridiques, économiques et financiers $d u$ développement écologique fonctionnant en Pologne doivent être perçus non seulement en direct à travers le prisme de régularité et cohérence formelle ainsi que l'efficacité économique et financière de l'ensemble du système, mais aussi il faut tenir compte du contexte plus large, soit de l'état du milieu naturel en Pologne et sa modification au cours de dix dernières années, l'état et le développement de l'infrastructure technique au service de la protection de l'environnement, la réalisation du principe de développement écologique dans notre pays et aussi l'intégration de la Pologne avec les structures de l'Union Européenne et l'adhesion notre pays de l'Union Européenne. Seulement une approche aussi large à ce problème permet d'effectuer une analyse honnête et une évaluation de l'efficacité du système entier et aussi programmer les modifications des instruments existants et créer les nouveaux instruments du développement écologique correspondants aux défis devant lesquels se place la politique écologique polonaise au seuil de XXI siècle.

Quand il s'agit de l'état du milieu naturel en Pologne et de l'infrastructure technique au service de sa protection, il faut constater que sue l'espace de dix dernières années nous sommes arrivés à une nette amélioration de l'état de tous les composants du milieu naturel c. à d. de l'eau, du sol et de l'air, en faisant toute une série des investissements d'infrastructure dans le domaine de gestion des eaux et eaux usées, gestion des déchets et la protection de l'air. Il ne faut pas oublier que ce grand effort d'investissement a été effectué dans la dernière décennie par l'Etat qui après plusieurs dizaines d'années des gouvernements totalitaires devait créer en parallèle les structures d'un régime démocratique, construire les collectivités locales, créer un système des finances publiques tout à fait nouveau, réaliser les reformes essentielles économiques et sociales. Il faut se rappeler aussi que les coúts de ces investissements étaient et sont presque en $95 \%$ financés des sources

Faculté de Droit de l'Université Catholique de Lublin. 
financières propres, nationales. Ce processus a obtenu une grande approbation sociale car contrairement à une opinion courante, la conscience écologique de la société polonaise est très haute. Les Polonais pareillement comme les citoyens des autres pays de l'Union Européenne, veulent bénéficier de l'eau pure, de l'air propre, veulent vivre dans un milieu naturel se trouvant au meilleur état possible. L'expression de cette conscience était l'élaboration et l'acceptation par le gouvernement déjà en 1991 de la I Politique Écologique de l'Etat qui était le premier document de ce type dans cette partie d'Europe et est devenu modèle pour les politiques écologiques des autres pays de la région. Il y a peu de pays en Europe, j'entends aussi les pays de l'Europe Occidentale, qui dans un aussi bref délai et avec les problèmes parallèles économiques, sociaux et de régime auraient effectué un progrès aussi signifiant dans la protection de l'environnement lequel fait part de la Pologne dans l'espace de la dernière décennie. Dès le début des années 90 nous observons une croissance extraordinaire des dépenses pour la protection de l'environnement, aussi bien dans le secteur privé que dans le rayon de l'économie locale. Les dépenses annuelles pour les investissements proécologiques ont augmenté de quelque fois dans les dernières années, atteignant en l'an 2003 le montant env. 8,7 mld PLN, ce qui constitue env. 1,5\% part dans le Produit National Brut.

Quand il s'agit de la structure des sources de financement des dépenses écologiques, elle est comme suit: les moyens propres des investisseurs et crédits (env. 46\%), moyens des fonds écologiques (env. $25 \%$ ), budgets des unités des collectivités locales (env. 20\%), budget d'État (env. 3\%), tous les moyens d'aide étrangère (env. 6\%) avec quoi on évalue que les sujets étrangers vont vendre en Pologne les services et technologies pro-écologiques pour une somme de 5-7 fois supérieure à toute aide étrangère.

Les résultats atteints par la Pologne dans la protection de l'environnement sont dans la grande mesure l'effet d'une application efficace des instruments économiques et financiers. Ils sont suivants:

- taxes pour l'utilisation du milieu et l'introduction des modifications dans ce milieu (y compris pour l'émission des polluants), nommés couramment taxes écologiques,

- pénalités pour la violation des exigences de protection de l'environnement, nommés couramment pénalités écologiques,

- subventions et prêts et crédits privilégiés pour les projets du domaine de protection de l'environnement obtenus des recettes des taxes et pénalités écologiques,

- différentiations et exemptions d'impôt.

Ce système était organisé par plusieurs années et toujours est en évolution, élargissant et rendant plus variable son offre. 
En plus on introduit à présent au droit polonais de protection de l'environnement les nouveaux instruments économiques tels que taxes sur produits et taxes de dépôt.

\section{Taxes écologiques}

Les taxes écologiques sont payées par les utilisateurs du milieu bien que leurs actions dans ce domaine soient conformes à la loi. Car ceci est une sorte de paiement pour l'utilisation, la pollution et la modification du milieu.

Les sujets économiques versent les taxes écologiques avant tout pour:

- l'introduction des polluants dans l'air,

- le puisage de l'eau étant la propriété de l'Etat,

- l'introduction des eaux usées dans les eaux étant la propriété de l'Etat ou au sol,

- le stockage des déchets,

- la coupe des arbres ou arbustes.

Ce sont les principales taxes écologiques avec lesquelles ont en principe à faire tous les sujets économiques profitant du milieu.

Le montant des taxes est calculé selon les tarifs en vigueur au jour d'utilisation du Milieu, fixés par le Conseil des Ministres par voie des arrêtés (annuels durant les dernières années), séparément pour chaque type d'utilisation du milieu et en fonction de la quantité et qualité des polluants introduits dans le milieu ou des modifications effectuées.

Les dispositions légales ne prévoient pas d'amortissement des taxes, des paiements à tempérament ni ajournement du délai de paiement.

Jusqu'à la fin de 1997 le montant de toutes sortes des taxes écologiques était calculé sous forme d'une décision "de taxe" par les organes d'administration: pour le puisage d'eau, évacuation des égouts, introduction des polluants dans l'air et le stockage des déchets - par le vodévode, et pour la coupe des arbres et arbustes - par l'organe de la commune.

A partir du 1 janvier 1998 ce système a été modifié pour les taxes de stockage des déchets (par la loi sur les déchets) et de l'introduction des polluants dans l'air (par la loi sur la modification de la loi sur la protection et formation de l'environnement).

Car on a introduit une obligation pour les sujets économiques à calculer leurs montants des taxes pour l'utilisation économique du milieu et l'introduction des modifications dans ce milieu et de les verser sur un compte de redistribution de l'office de vodévodie, de ressort par rapport au lieu d'utilisation du milieu, sans décision du vodévode.

Par la loi du 24 juillet 1998 sur la modification des certaines lois définissant les compétences des organes d'administration publique - en 
liaison avec la réforme du régime du pays, à partir du 1 janvier 1999 le maréchal de vodévodie a repris du vodévode les compétences dans le domaine de cumul et exécution des taxes écologiques.

Les taxes pour l'émission des polluants dans l'air sont déterminées pour 62 types de substances polluantes divisées en trois groupes. Les taux les plus élevés ont été fixés pour les polluants toxiques tels que l'essence, amiante, chrome, nickel.

L'évacuation des égouts est chargée par les taxes fixées pour six indices. Les taxes sont fixées aussi pour le puisage des eaux de surface et souterraines. Les taux pour les utilisateurs particuliers sont différentiés très fort en fonction des secteurs d'économie et des vodévodies.

Les taxes imposées pour le stockage des déchets comprennent toutes les sortes des déchets qui sont placés dans le classement des déchets. On a adapté quatre niveaux des taux en fonction du degré de nuisance des déchets.

La loi sur les déchets qui est entrée en vigueur le 1 janvier 1998 et suivant la nouvelle loi sur les déchets du 27 avril 2001 a élargi le système des taxes pour le stockage des déchets en introduisant à côté des taxes pour le placement des déchets en dépôt en même temps les taxes pour le temps de leur stockage. Ceci a pour but renforcer le rôle stimulant des taxes écologiques dans le domaine de limitation de la quantité des déchets stockés dans les dépôts et augmenter leur utilisation économique.

La taxe pour la coupe des arbres et arbustes, administrée localement, dans les communes, est fixée en fonction d'espèce de l'arbre et du périmètre de son tronc.

\section{Pénalités écologiques}

Les pénalités écologiques sont pénalités pécuniaires, payées par les sujets économiques pour le non-respect des exigences de la protection de l'environnement. Contrairement aux taxes qui sont à la charge des coüts de production, les pénalités sont perçues du revenu après imposition et par le même doivent être instrument d'action plus stimulant que les taxes.

Les pénalités sont infligées, à l'exclusion des pénalités pour la coupe des arbres et arbustes, par les inspecteurs de protection d'environnement au niveau de vodévodie, fonctionnant jusqu'à la fin de 1998 comme organes d'administration spéciale, et à partir du 1 janvier 1999 dans le cadre d'administration associée sous couvercle du vodévode.

L'application d'une pénalité pécuniaire est causée par:

- infraction des dispositions de la décision sur l'émission admissible des polluants dans l'air - quant à la nature ou à la quantité des substances, 
- transgression des conditions définis dans le permis légal d'eau définissant les conditions admissibles d'évacuation des égouts ou absence d'un tel permis,

- infraction de la décision sur le niveau du bruit admissible - quant au niveau du bruit pénétrant dans le milieu,

- stockage des déchets dans un endroit n'étant pas destiné à ce but ou en non-conformité aux exigences définies par la décision de l'organe compétent pour les affaires de surveillance de construction dans le permis de construire du dépôt des déchets.

Dans le domaine du système des pénalités bien fonctionne le mécanisme d'ajournement des paiements pour une période allant à 5 ans, dans le cas de réalisation par les unités punies des investissements proécologiques, dont la fin permet d'éliminer la cause de calcul des pénalités. Dans le cas de fin des tels investissements à terme, le montant de la pénalité ajournée est diminué de la grandeur des moyens propres de l'établissement subis pour leur réalisation. Dans la majorité des cas cette pénalité est pratiquement amortie. Les pénalités ajournées concernant les déchets, dans le cas de réalisation à terme du projet, sont amorties en totalité, indépendamment de l'importance des moyens propres d'établissement engagés. On évalue que dans les dernières années environ $80 \%$ d'établissements bénéficiant des remises des délais réalise les investissements étant la base d'application de cet instrument. Dans le cas de non-réalisation à terme de la mission, l'établissement est tenu de payer la pénalité ajournée avec son augmentation comme sanction.

Le système d'infliger les pénalités liées avec la possibilité de leur ajournement sert à forcer les actions d'investissement pro-écologique et pas l'obtention des moyens financiers.

Les recettes des taxes et pénalités écologiques constituent une source d'alimentation du Fonds National de Protection d'Environnement et de l'Économie Hydraulique de 16 fonds de protection d'environnement et de l'économie hydraulique au niveau des vodévodies, des fonds de protection d'environnement et de l'économie hydraulique au niveau des districts et communes, permettant le fonctionnement en Pologne d'un large système de subventions des projets pro-écologiques, sous forme des subventions et des prêts et crédits privilégiés.

\section{Différentiations et exemptions d'impôt}

Les instruments économiques présents dans l'économie polonaise, tels que les différentiations et exemptions d'impôt, n'avaient pas jusqu'à là de grand impact sur la réalisation des investissements écologiques et 
modification du comportement de la société. Ces instruments dans la plupart des cas n'étaient pas constitués en pensant à la protection de l'environnement, alors ne la concernaient que dans un cadre limité.

La possibilité de déduction des dépenses d'investissement pour la protection de l'environnement de la base imposable du revenu était liée aux dispositions générales dans ce domaine et n'avait pas de caractère préférentiel.

A partir de l'an 2000 dans le cadre de la reforme du système fiscal en Pologne, on a liquidé toutes les facilités d'investissement dans l'impôt sur le revenu des personnes morales, et on prévoit une solution semblable dans les dispositions concernant l'impôt sur le revenu des personnes physiques.

Une certaine action de stimulation concerne l'accise introduite pour les carburants liquides. Car elle est un peu inférieure pour l'essence sans plomb et des gasoils sans souffre. En plus dès la moitié 1995 on a introduit l'accise pour les emballages en matières plastiques, en appliquant une facilité partielle pour les emballages en matières moins nuisibles pour le milieu.

Aussi dans la petite mesure la différentiation de la taxe TVA donne la préférence aux marchandises et services selon le critère de protection de l'environnement.

Un des instruments économiques dans la protection de l'environnement sont les tarifs de douane préférentiels pour l'importation des certains dispositifs et appareils de mesure servant à la protection de l'environnement des pays de l'Union Européenne.

Dans la loi concernant l'impôt sur le revenu des personnes morales et physiques on a introduit les exemptions de l'impôt sur le revenu d'une partie des revenus de l'activité économique dans laquelle on utilise les déchets fabriqués sur le terrain de la République de Pologne, fixé dans un tel rapport, dans lequel pour la dernière année fiscale reste la valeur des déchets utilisés dans la valeur globale des matières premières et des déchets utilisés dans le processus de production.

Le Ministre des Finances en accord avec le Ministre de l'Environnement a défini, par voie d'un arrêté les types des déchets dont l'utilisation autorise à l'exemption ci-dessus et les principes de fixation de la valeur des déchets utilisés dans le processus de production.

En plus dans le cas de réalisation des investissements de domaine de protection d'environnement on a le droit aux déductions d'amortissements de la valeur initiale de l'actif immobilisé et des immobilisations incorporelles. 


\section{Subventions ainsi que les pręts et crédits privilégiés pour les projets dans la protection de l'environnement}

Comme j'ai mentionné les résultats dans la protection de l'environnement atteints au cours de dernières années sont en grande mesure l'effet de création et fonctionnement efficace en Pologne d'un système intégré de financement de la protection de l'environnement, appuyé avant tout sur les fonds écologiques et sur les autres sources de financement co-agissant avec eux, parmi lesquels il faut citer avant tout EKOFUNDUSZ, gérant les moyens d'ainsi nommée éco-conversion, soit provenant de la conversion de la dette étrangère polonaise en projets pro-écologiques en Pologne. Le fonctionnement de ce système consiste à assister par les prêts et crédits privilégiés ainsi que subventions, les moyens des propres investisseurs. Ils sont complétés par les moyens provenant du budget central et des budgets locaux, des différentes sortes des fondations écologiques, banques commerciales, dont de la Banque de Protection de l'Environnement et de l'aide étrangère dans un sens très large.

Les subventions initiales très répandues sont remplacées de plus en plus par les prêts privilégiés et complément aux crédits bancaires. Aussi le processus de coopération et de co-action des fonds écologiques et des banques devient de plus en plus large. Dans le document AGENDA 2000 la Commission Européenne a annoncé la constitution d'un nouveau mécanisme financier, destiné à soutenir les pays associés de l'Europe Centrale et de l'Est dans le processus d'adaptation de leurs systèmes juridiques et économies aux standards de l'Union Européenne. A partir de l'an 2000 les fonds communautaires spéciaux fonctionnent, dont le Fonds ISPA, ciblé sur l'adaptation de l'infrastructure technique dans le domaine de la protection de l'environnement et du transport des pays associés aux standards de l'Union Européenne. A partir de 1 mai 2004 la Pologne comme le membre de l'Union Européenne peut utiliser les Fonds Structurels, entre autres, pour financer les investissements d'infrastructurels dans le domaine de la protection de l'environnement.

Du Fond Européenne de Developpement Regional peuvent être financé les projets $d^{\prime}$ investissements suivants:

- les stations urbaines d'épuration des égouts (unitaires plus grandes ou plus petites en paquet créant le programme de traitement du bassin de fleuve donné),

- les systèmes d'égout de ville,

- les systèmes d'alimentation en eau et du traitement d'eau potable,

- la modernisation et extension des systèmes urbains de chauffage, liée à la liquidation d'ainsi nommée basse émission, 
- les investissements pro-écologiques dans les systèmes de transport urbain,

- la construction, extension ou modernisation des dépôts des déchets communaux,

- les systèmes de recyclage et utilisation des déchets communaux (triage, engraissement etc.)

- les systèmes de collecte et utilisation des déchets nuisibles et dangereux.

Chaque investissement présenté à un cofinancement du FEDR doit répondre rigoureusement aux normes et standards techniques et écologiques en vigueur en Union Européenne.

Un autre grand fonds avant accès proposé par l'Union Européenne aux pays sollicitant de devenir membres a été le Fonds SAPARD, servant à donner appui à l'agriculture et au développement des terrains ruraux. Sur la liste des projets lesquels ont été financé du Fonds SAPARD sont avant tout les missions visant une amélioration de l'efficacité du marché agricole, amélioration des standards de qualité et de santé des produits agricoles ainsi que la création des nouvelles places du travail sur les terrains ruraux. Néanmoins les moyens de ce Fonds peuvait partiellement, au moins indirectement, assister la protection de l'environnement pex. Dans le cadre du développement et amélioration de l'infrastructure rurale, investissements dans seules exploitations agricoles ou programmes de boisement des terres en friche.

Comme j'ai déja ecrit uu moment d'accès à l'Union Européenne la Pologne a perdu la possibilité de bénéficier du Fonds ISPA et SAPARD, mais notre pays a gagné l'accès aux fonds structurels de l'Union bien plus importants. Ces fond vont jouer le rôle d'un instrument fort d'aide assurant la destination des grands moyens financiers, entre autres pour la protection de l'environnement et assistant les missions réalisées dans ce cadre par les collectivités locales et les sujets économiques.

\section{Directions des changements successifs du système de financement de la protection d'environnement en Pologne}

L'économie polonaise a toujours devant elle des immenses tâches à remplir dans la protection de l'environnement lesquelles résultent aussi bien du besoin de construction et de la modernisation de l'infrastructure technique de la protection de l'environnement comme aussi des conditions de l'Union Européenne.

Les instruments économiques peuvent jouer un rôle essentiel dans la réalisation de ces tâches. Le système des instruments économiques utilisés 
dans la gestion de la protection de l'environnement fonctionnant actuellement en Pologne est surtout basé sur le système des taxes pour l'émission des polluants dans le milieu et des pénalités pour le dépassement des exigences de la protection de l'environnement. On évalue que ce système a bien passé son examen dans les premières années des changements économiques et politiques en Pologne, en contribuant à une nette amélioration de l'état d'environnement dans notre pays.

Avec le développement de l'économie de marché les modifications de ce système sont cependant nécessaires, elles doivent permettre une croissance des stimulants provoquant les comportements pro-écologiques des sujets économiques et de la société entière ainsi que permettre une croissance des dépenses pour la protection de l'environnement.

Dans ce but on propose d'introduire à la législation polonaise de la protection de l'environnement les taxes écologiques de produit et de dépôt par la loi concernant les obligations des entrepreneurs dans le cadre de gestion des certains déchets et sur la taxe de produit et de dépôt. La loi sur les taxes écologiques de produit et de dépôt est entrée en vigueur le 1 janvier 2002. Sous les taxes de produit on entend les charges financières ajoutées aux prix des produits, lesquels utilisés en masse et dispersés constituent une nuisance pour le milieu dans la phase de production, consommation ou stockage après production. Le but de l'application de la taxe de produit est le cumul des moyens financiers pour solutionner une tâche écologique définie et en même temps la stimulation des comportements correspondants des consommateurs et entrepreneurs.

Cependant les dépôts écologiques se sont les charges financières ajoutées au prix des produits écologiquement nuisibles, remboursés au moment de transfert des produits pour le recyclage, neutralisation ou stockage propre sous biais écologique. Le but principal d'application des dépôts écologiques a été alors la stimulation du retour du produit usé, pour permettre son recyclage ou un stockage en sécurité.

Les taxes sont imposées sur les produits, lesquels sont déjà soumis aux charges similaires dans plusieurs pays et dont l'application $\mathrm{y}$ a conduit aux effets écologiques favorables et/ou fiscaux. Aussi leur montant est proche aux taux adaptés dans les autres pays. Leur mise en suvre n'a constitué donc pas une expérience. Mais dans les conditions polonaises s'a été un tournant essentiel, car à part une petite accise pour les emballages en plastique introduite en 1995, il n'y avait jusqu'à là des instruments ayant caractère fiscal strictement lié à la nuisance écologique des produits. 


\section{Instruments économiques et financiers du développement écologique dans le contexte d'intégration de la Pologne avec l'Union Européenne}

L'objectif de base, stratégique de la politique étrangère polonaise a été un accès à l'Union Européenne. Dans le processus des négociations, dont l'objet a été notre qualité de membre dans l'Union Européenne "l'environnement" a été un des sujets les plus importants et difficiles. Afin de satisfaire aux exigences de l'Union Européenne la Pologne a été obligé d'effectuer une transposition à l'ordre légal national de plus de 170 actes du droit communautaire secondaire dans le domaine du droit concernant la protection de l'environnement. En premier lieu a été mis en śuvre ces actes légaux lesquels ont l'influence sur le fonctionnement du marché homogène. Conformément à la position polonaise des négociations, vu les coúts élevés des programmes d'adaptation, et aussi les résultats sociaux négatifs possibles, causés par une mise en śuvre trop rapide de tous les actes juridiques de l'Union Européenne (la croissance de l'inflation, élimination du marché des sujets économiques plus faibles entraînant une hausse du chômage etc.), par rapport aux certains actes juridiques la partie polonaise a demandé les périodes transitoires. On a admis que dans l'espace „Environnement” l'arrivée à la conformité aux exigences de l'Union Européenne sera faite en 3 étapes. Ces étapes comprend les buts suivants:

- la transposition au droit polonais de l'acquis communautaire;

- les changements institutionnels;

- la modernisation et adaptation du réseau national de monitoring de l'environnement aux exigences de l'Union Européenne;

- l'élaboration des programmes détaillés de la qualité des eaux;

- la vérification des programmes existants et créations des nouveaux programmes de la gestion des déchets;

- la réalisation des investissements liés à la mise en śuvre de ces actes légaux de l'Union Européenne par rapport auxquels la Pologne ne sollicite pas les périodes transitoires;

- le réalisation des investissements d'infrastructure dans les agglomérations, conformément aux programmes existants de gestion des déchets;

- le commencement des investissements dans le domaine de mise en ordre de la gestion des égouts et d'alimentation des plus grandes agglomérations urbaines en eau potable;

- la réalisation des investissements suivants dans les villes et collectivités locales:

- les stations urbaines d'épuration des égouts (unitaires plus grandes ou plus petites en paquet créant le programme de traitement du bassin de fleuve donné), 
- les systèmes d'égout de ville,

- les systèmes d'alimentation en eau et du traitement d'eau potable,

- la modernisation et extension des systèmes urbains de chauffage, liée à la liquidation d'ainsi nommée basse émission,

- les investissements pro-écologiques dans les systèmes de transport urbain,

- la construction, extension ou modernisation des dépôts des déchets communaux,

- les systèmes de recyclage et utilisation des déchets communaux (triage, engraissement etc.)

- les systèmes de collecte et utilisation des déchets nuisibles et dangereux.

Avec la transposition des dispositions communautaires à la législation polonaise est liée la nécessité de mise en śuvre de toutes leurs exigences afin d'assurer d'atteindre le niveau déterminé de la protection de l'environnement et de l'état convenable de ses éléments particuliers c. à d. de l'air, du sol et des eaux etc. Le processus de mise en śuvre est lié à la nécessité d'effectuer une série d'investissements ayant pour but une amélioration de l'état du milieu naturel et l'acquis des standards communautaires. Les dépenses d'investissement globales cumulées du paquet des projets pro-écologiques sont évalués à un total environ 30-35 mld euros. Les plus importants seront les investissements dans les espaces suivantes: de la protection d'eau et de la gestion des eaux et des eaux usées, gestion des déchets, protection de l'air et prévention des polluants industriels.

Les coűts d'investissements devront être subis aussi bien par les sujets publiques que privés. A présent on évalue qu'en Pologne on dépense par an 2,2 mld euros pour les investissements pro-écologiques. La différence de bilan entre la somme de demande en capital indispensable au financement des investissements, et la somme des moyens financiers accessibles est évalué pour un montant entre 2,1 et 3 mld euros. La lacune financière sera particulièrement visible dans les deux ou trois premières années de mise en śuvre des dispositions de l'Union Européenne. Bien que le système légal, financier et économique fonctionnant en Pologne étant la base de la politique écologique de l'Etat soit intérieurement cohérent et effectif, il semble que les possibilités de génération des moyens financiers par les instruments existant ont atteint un seuil maximal. Alors pour cela on ne pourra pas remplir la lacune financière mentionnée ci-dessus seulement s'appuyant sur les sources existantes et les instruments de financement des investissements proécologiques. Les fonds supplémentaires devront être obtenus de l'introduction des nouveaux instruments légaux, financiers et économiques (entre autres les taxes de produit et de dépôt mentionnées, 
et les moyens d'aide étrangère d'avant accès et de cohésion, la charge plus grande des utilisateurs par les frais de la protection de l'environnement). Le poids du financement et des investissements proécologiques repose aussi bien sur les sujets publics (pex. Les collectivités locales) comme sur les sujets privés (entrepreneurs). Par rapport au premier groupe des sujets il faut observer le phénomène de croissance du niveau d'endettement des collectivités locales et d'arrivée par des nombreuses entre elles aux limites hautes d'endettement et de service d'endettement désignés par la loi sur les finances publics. La conséquence de cela est l'absence de possibilités d'assurer le financement de tous les coüts d'investissement et l'ajournement ou la prolongation de la période de réalisation des projets particuliers. Par suite pour les entrepreneurs réalisant les investissements pro-écologiques, et aussi pour les institutions financières desservant cet espace de l'activité économique, la loi sur les conditions d'admission et surveillance de l'aide publique pour les entrepreneurs a une grande importance. A présent les travaux des experts sont en cours, dont l'objet est d'établir avec précision les conséquences légales et financières de cette loi pour le secteur des investissements écologiques.

Tenant compte de l'état légal et réel signalé plus haut on peut constater qu'il existe un besoin urgent de la réponse à la question: de quelle manière avec les instruments légaux, économiques ou financiers existants ou nouveaux combler la lacune dans le système de financement des investissements pro-écologiques pour ne pas permettre un retard du programme de mise en suvre des normes communautaires du droit écologique et d'atteindre les standards écologiques de l'Union Européenne. Le retard potentiel peut avoir un impact défavorable pour atteindre les objectifs du développement équilibré social, économique et écologique dans notre pays.

Tenant compte de ceci, le Ministère de la Protection d'Environnement a commencé avec beaucoup d'avance les actions visant la création d'un paquet des nouveaux instruments légaux, économiques ou financiers du développement écologique et la modification des anciens. A part l'introduction des taxes écologiques décrites avant, de concert avec le Fonds National de Protection de l'Environnement et de la Gestion des Eaux (NFOŚiGW), Ekofundusz, Banque de Protection de l'Environnement et la Bourse des Valeurs (GPW) on continu les travaux sur le concept des nouveaux instruments financiers. On considère une intensification de l'activité de capital NFOŚiGW et la proposition aux bénéficiaires des nouvelles solutions dans ce domaine. Car NFOŚiGW doit jouer le rôle d'investisseur passif de capital dans les sociétés communales et assister de cette façon les investissements pro-écologiques d'infrastructure réalisés par les collectivités locales ou les sujets économiques dépendant d'eux. De 
concert avec GPW on a commencé les actions créant le marché des obligations écologiques, lesquelles seraient une source supplémentaire du financement des investissements écologiques. Il sera aussi d'importance extraordinaire pour la Pologne d'augmenter les moyens financiers destinés aux investissements écologiques dans le cadre de l'éco-conversion par les gouvernements des pays créanciers de la Pologne. Dans ce but en commun avec Ekofundusz on engagera les actions visant les négociations pour augmentation des moyens pour éco-conversion. L'éco-conversion de la dette polonaise est favorable sur le plan financier aussi bien pour la Pologne qu'aux pays - créanciers. Notre pays obtient alors les moyens supplémentaires pour les investissements écologiques, et les pays créanciers bénéficient par l'achat des produits et services de leurs entreprises nationales desservant les investissements particuliers (la valeur de cette vente dépasse quelque fois les montants de l'éco-conversion). On estime que l'accès de l'Allemagne, Autriche et Canada à l'éco-conversion aurait apporté à l'écologie polonaise environ $1 \mathrm{mld}$ USD au cours de 10 ans. Sont en cours les travaux pour la création des bases légales et d'organisation pour mettre en marche en Pologne le négoce des unités de réduction de l'émission $\mathrm{CO} 2$ et $\mathrm{SO} 2$ ainsi que de l'utilisation effective du mécanisme joint implemtation. L'application en Pologne des éléments de réduction de l'émission prévus au Protocole de KYOTO à la Convention Climatique est évaluée comme favorable économiquement dans le contexte de la dynamique actuelle du développement de notre économie et industrie. On a commencé aussi les travaux ayant pour but l'élaboration de la taxe écologique sur les carburants. La charge des carburants particuliers par la taxe écologique de quelques groszys qui pourrait être le revenu des fonds écologiques, avec l'impulse d'inflation minimale, aurait apporté au moins 1,5 mld PLN par an.

Il y est extrêmement important d'utiliser effectivement les moyens financiers provenant de l'aide étrangère, c. à d. avant tout des fonds structurels et Fond Cohesion de l'Union Européenne, Banque Mondiale, des conventions bilatérales. Les fonds principals d'aide dans le domaine de la protection de l'environnement sont actuellement fonds structurels et Fond Cohesion de l'Union Européenne. Dans la première année du fonctionnement de ces fonds la partie polonaise compte que la Commission Européenne va octroyer $100 \%$ des moyens financiers possibles à percevoir dans l'an 2004, alors les fonds pourraient complètement utilisé.

A présent les moyens provenant de fonds structorels et du Fond Cohesion constituent la plus grande partie de l'aide communautaire pour la Pologne dans le domaine de la protection de l'environnement. Pour les années 2004-2006 l'Union Européenne a destiné plus de 14 mld euros pour la coonfincement sous forme de ces fonds. 
Tous les projets appuyés par ces fond doivent être effectifs économiquement. Il faut se rappeler que cela n'est pas équivalent à la rentabilité financière. L'analyse des frais sociaux et des profits (CBA) doit démontrer le profit net de la réalisation de l'investissement pour la société.

Les projets qui ont la chance d'obtenir un appui des moyens fonds structurels ou Fond Cohesion ne doivent pas forcement être rentables financièrement sans subventions des sources publiques. Cependant avec les subventions les indices économiques doivent franchir le seuil de rentabilité, ce qui est condition nécessaire pour que le projet puisse être réalisé par l'investisseur. Il faut aussi prouver la fluidité financière du projet dans la période d'exploitation ou documenter la capacité d'en assurer. Une rentabilité financière trop haute du projet entraînera un refus ou une diminution de la subvention car cela va signifier que le projet peut être financé des sources commerciales.

La Pologne se trouve maintenant devant une grande chance du développement économique créée par une adhesion de l'Union Européenne, en plus nous pouvons bénéficier des nouvelles possibilités apportées par l'application des principes du développement social et économique équilibré avec respect et bonne utilisation du milieu naturel. L'entrée de la Pologne sur la voie du développement écologique est une tâche pour les années à venir aussi bien pour l'autorité publique que pour les partenaires sociaux.

\section{Prawne i finansowe instrumenty ekorozwoju w Polsce}

\section{STRESZCZENIE}

Funkcjonujące $w$ Polsce prawne, ekonomiczne oraz finansowe instrumenty polityki ekologicznej należy postrzegać nie tylko wprost, poprzez pryzmat poprawności i spójności formalnej oraz efektywności ekonomiczno-finansowej całego systemu, ale również należy mieć na uwadze szerszy kontekst, tj. stań środowiska naturalnego $\mathrm{w}$ Polsce $\mathrm{i}$ jego zmiany na przestrzeni ostatnich dziesięciu lat, stan i rozwój infrastruktury technicznej służącej ochronie środowiska, realizację zasady ekorozwoju w naszym kraju, a także integrację Polski ze strukturami Unii Europejskiej. Tylko takie szerokie ujęcie problemu pozwala na dokonanie rzetelnej analizy i oceny efektywności całego systemu, a także na zaprogramowanie modyfikacji istniejących oraz stworzenia nowych instrumentów ekorozwoju odpowiadających wyzwaniom, przed którymi polska polityka ekologiczna staje na początku XXI wieku.

Jeżeli chodzi o stan środowiska naturalnego w Polsce oraz infrastruktury technicznej służącej jego ochronie, to należy stwierdzić, że na przestrzeni 
ostatnich dziesięciu lat udało się osiągnąć znaczącą poprawę stanu wszystkich elementów środowiska naturalnego, tj. wody, gleby i powietrza, dokonując szeregu inwestycji infrastrukturalnych $w$ zakresie gospodarki wodno-ściekowej, gospodarki odpadowej oraz ochrony powietrza.

Osiągane przez Polskę wyniki w ochronie środowiska są w dużej mierze efektem skutecznego stosowania instrumentów ekonomicznych oraz finansowych. Należą do nich:

- opłaty za korzystanie ze środowiska i wprowadzanie w nim zmian (w tym za emisję zanieczyszczeń),

- opłaty produktowe i opłaty depozytowe,

- kary za naruszanie wymagań ochrony środowiska,

- dotacje oraz preferencyjne pożyczki i kredyty dla przedsięwzięć z zakresu ochrony środowiska ze środków uzyskiwanych z wpływów z opłat i kar ekologicznych,

- zróżnicowania i zwolnienia podatkowe.

Polska gospodarka nadal ma przed sobą do spełnienia ogromne zadania w ochronie środowiska, które wynikają zarówno $z$ potrzeby budowy i modernizowania infrastruktury technicznej ochrony środowiska, jak $\mathrm{i}$ też $\mathrm{z}$ warunków członkostwa Polski w Unii Europejskiej. Istotna rolę $w$ realizacji tych zadań muszą odegrać instrumenty prawno-finansowe. Funkcjonujący obecnie $w$ Polsce system instrumentów prawno-finansowych wykorzystywanych w zarządzaniu ochroną środowiska oparty jest przede wszystkim na systemie opłat za emisję zanieczyszczeń do środowiska i kar za przekraczanie wymagań ochrony środowiska.

Wraz z rozwojem gospodarki rynkowej konieczne są jednak zmiany tego systemu, które powinny pozwolić na zwiększenie bodźców stymulujących proekologiczne zachowania podmiotów gospodarczych i całego społeczenstwa oraz umożliwić wzrost wydatków na ochronę środowiska.

Polska stoi obecnie przed wielką szansą dynamicznego rozwoju, jaka stwarza członkostwo w Unii Europejskiej. Wiele korzyści może przynieść stosowanie zasad zrównoważonego rozwoju społecznego i gospodarczego z poszanowaniem i właściwym wykorzystaniem środowiska naturalnego. Utrzymanie przez Polskę stabilnego kursu na drodze ekorozwoju jest zadaniem na najbliższe lata zarówno władzy publicznej, jak i też przedsiębiorców i partnerów społecznych. 